\title{
Cascade photo production at CLAS
}

\author{
John T. Goetz* \\ Ohio University \\ E-mail: goetz@jlab.org
}

\section{Ken Hicks}

Ohio University

E-mail: hicks@jlab.org

\begin{abstract}
The famous discovery of the $\Omega^{-}$in 1964 put the quark model on firm ground and since then a lot of effort has been spent on mapping out the baryonic and mesonic states. Over the following decades, many excited baryons with light quarks (up, down and strange) have been measured, but by most predictions, only a small percentage of those expected have been found. In this talk, I will discuss a newly developing technique using an (unflavored) photon beam to excite protons to doubly-strange "Cascade" $(\Xi)$ states. Advantages of such an experiment and associated difficulties will be presented, along with recent results from the CLAS detector at Jefferson Lab in Virginia.
\end{abstract}

XV International Conference on Hadron Spectroscopy

4-8/11/2013

Nara, Japan

${ }^{*}$ Speaker. 


\section{Cascade Hyperons}

Using QCD's powerful symmetry of strong-coupling flavor independence, one would predict that for every $\mathrm{N}^{*}$ and $\Delta^{*}$ resonance, there would be a corresponding $\Xi^{*}$ state. In addition, quark-models[1], chiral-dynamic models[2] and algebraic models[3] all predict several dozens of cascade states to exist and recent results from lattice $\mathrm{QCD}[4]$, which in principal accounts for dynamic effects, expects similar behavior. However, only a handful of cascade states have been observed with any certainty despite being narrower than nonstrange baryons - a property which makes them relatively easy to measure once produced.

Virtually all evidence for $\Xi^{*}$ resonances come from measuring the decay particles directly in hadron-production experiments such as $\mathrm{K}^{-} \mathrm{p} \rightarrow \Xi^{-} \mathrm{K}^{+}$and $\Sigma^{-} \mathrm{p} \rightarrow \Xi^{0} \mathrm{pK}^{-}$. With the CLAS detector at Jefferson Laboratory in Virginia, USA, we are studying the cascade states through photoproduction:

$$
\gamma \mathrm{p} \rightarrow \Xi^{*-} \mathrm{K}^{+} \mathrm{K}^{+},
$$

where we detect the two kaons and observe the cascade through missing mass. The two primary channels for photo-production involve producing a massive $\mathrm{Y}^{*}$ which decays to the cascade via kaon-exchange or through an s-channel $\mathrm{N}^{*}$ decaying by kaon emission. These and higher-order production mechanisms in relation to the experiment discussed in the next section are discussed in Ref. [5].

\section{Recent Results with the CLAS Detector}

The g12 experiment at Jefferson Laboratory in Virginia, USA used a tagged, realphoton, bremsstrahlung-spectrum beam on a liquid hydrogen target with the CLAS detector. The total photon luminosity, produced from a 60-65 nA electron beam, was approximately $68 \mathrm{pb}^{-1}$ and the peak photon energy was $5.4 \mathrm{GeV}(\sqrt{s}=3.3 \mathrm{GeV})$. Roughly 20k ground-state $\Xi^{-}(1320)$ were measured along with $5 \mathrm{k} \Xi^{-}(1530)$. These data were analyzed $[6]$ with several different kinematic and fiducial constraints (e.g. with and without requiring a detected proton) and no evidence of higher mass cascades state was observed. The strongest signal in previous (hadron-production) data came from the $\Xi(1820)$ with a width of $24 \mathrm{MeV}$ and we have estimated the upper-limit to less than $1.1 \mathrm{nb}$ with a $90 \%$ confidence (see Fig. 1).

Currently, we are finalizing the absolute scaling of the differential cross section of the two ground state cascades which covers nearly-complete angular coverage (see Fig. 2) from threshold to $\sqrt{s}=3.3 \mathrm{GeV}$. It is interesting to note that we see a leveling off of the excitation function (Fig. 3) of the $\Xi^{-}$(1320) from 4 to $5.5 \mathrm{GeV}(\sqrt{s}=2.8$ to $3.3 \mathrm{GeV}$ ). This indicates that with the increase in energy to $12 \mathrm{GeV}$ at Jefferson Lab, and with the CLAS12 and Glue-X detectors, we may continue the search for these high-mass cascade states in the near future.

\section{References}

[1] Capstick et al, Nucl Phys, 45 (Suppl 2): S241-S331 


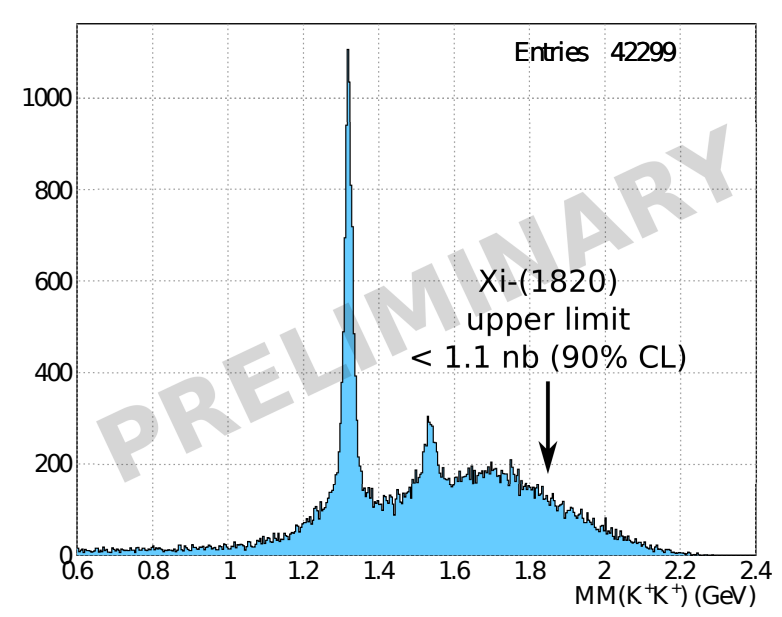

Figure 1: Missing mass off $\mathrm{K}^{+} \mathrm{K}^{+}$where a final state proton was detected in CLAS.

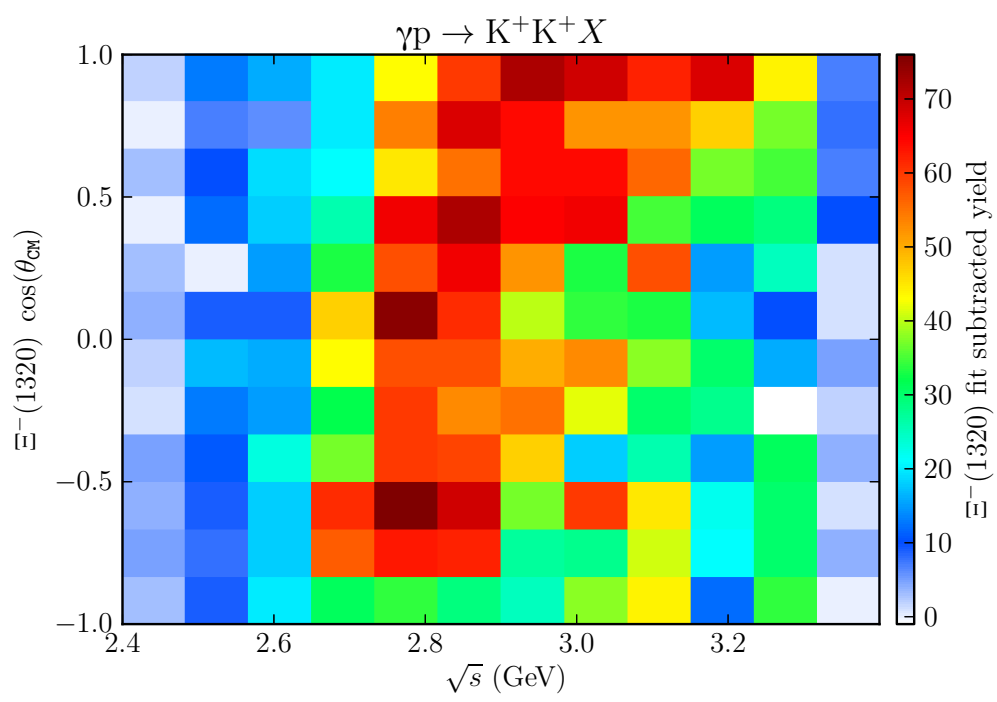

Figure 2: Differential yield of the ground state $\Xi^{-}(1320)$ calculated from missing mass off $\mathrm{K}^{+} \mathrm{K}^{+}$ showing near-complete angular coverage of the cascade in the center of mass frame.

[2] Glozman et al, Phys Rep, (268):263-303, 1996

[3] Bijker et al, Annals of Phys, 284(1):89-133, 2000

[4] R. Edwards et al, Phys Rev D 87, 054506 (2013)

[5] K. Nakayama, Yongseok Oh, and H. Haberzettl 2007 J. Phys.: Conf. Ser. 69012023

[6] J. T. Goetz. $\Xi$ Hyperon Photoproduction from Threshold to $5.4 \mathrm{GeV}$ with the CEBAF Large Acceptance Spectrometer. PhD thesis, University of California Los Angeles, 2010 


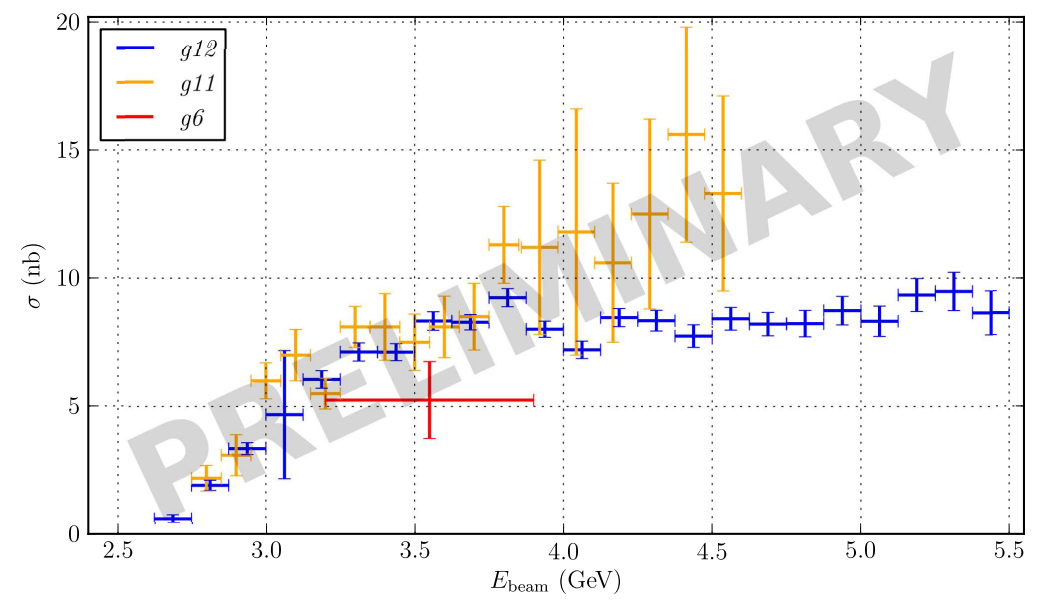

Figure 3: Excitation of the ground state $\Xi(1320)$ from threshold to $5.5 \mathrm{GeV}$ showing the comparison with previous CLAS experiments g11 and g6. 